\title{
La situación de las personas con capacidad intelectual límite en España ${ }^{1}$
}

\author{
The situation of people with borderline \\ intelligence in Spain
}

\section{Resumen}

Este artículo presenta los resultados principales de un estudio de carácter fundamentalmente descriptivo sobre la situación actual de las personas con capacidad intelectual límite en 20 I 5 . El estudio se centra en describir la realidad demográfica y social de las personas con capacidad intelectual límite, sus necesidades de apoyo y extraer propuestas estratégicas hacia una inclusión social efectiva. Metodológicamente, de acuerdo al nivel de análisis, se optó por técnicas cuantitativas (análisis de fuentes estadísticas) y cualitativas (entrevistas semiestructuradas a un grupo de personas con capacidad intelectual límite, familias y profesionales). Entre sus principales conclusiones, las personas con capacidad intelectual límite y sus familias demandan una visibilización de sus necesidades de apoyo para una inclusión social efectiva, siendo clave el apoyo para favorecer una vida autónoma e independiente. Las propuestas y buenas prácticas aportan elementos para un modelo de atención individualizada, al mismo tiempo fundamentada en una participación activa en la comunidad.

\section{Palabras clave}

Capacidad intelectual límite, inclusión social, derechos humanos, servicios, apoyos.

\begin{abstract}
This article presents the result of an investigation on the situation of people with borderline, in 20I 5. It is a descriptive study on the social reality of people with borderline intelligence, their needs for support and strategic proposals for an effective social inclusion. Methodologically quantitative techniques, such as analysis and exploitation of statistic sources and qualitative techniques (interviews with open-ended questions) to a group of people with borderline intelligence, family, and professionals were used. One of the main conclusions is that people with disabilities and their families demand a visibility of their needs of support, fundamental for an effective social inclusion and for encouraging an autonomous and independent life. The proposals and best practices provide elements for a person-centred model of care and an active community participation.
\end{abstract}

\section{Keywords}

People with borderline intelligence, social inclusion, human rights, services, supports.

\author{
Agustín Huete García \\ <ahueteg@intersocial.es> \\ Universidad de Salamanca. Intersocial
}

\section{Pilar Pallero Soto}

<pilarpallero@intersocial.es>

Intersocial

\author{
Para citar: \\ Huete, A. y Pallero, M. (20I6): \\ "La situación de las personas \\ con capacidad intelectual límite \\ en España", Revista Española de \\ Discapacidad, 4 (I): 7-26.
}

Doi: <http://dx.doi.org/IO.5569/23405 I04.04.OI.OI>

Fecha de recepción: 2I-02-20I6

Fecha de aceptación: 25-05-20I6

I. Este artículo es un extracto de un estudio más amplio titulado "Estudio sobre la situación de las personas con capacidad intelectual límite” publicado por el Ministerio de Sanidad, Servicios Sociales e Igualdad en 2015 . 


\section{Introducción}

Las personas con capacidad intelectual límite vienen demandando en los últimos años, fundamentalmente a través de las entidades que les representan, un reconocimiento social y, especialmente, un reconocimiento de derechos en las políticas y actuaciones de las diferentes administraciones públicas. Precisamente, el Observatorio Estatal de la Discapacidad se ha hecho eco de esta necesidad, impulsando la realización de un estudio sobre situación y necesidades de esta población, que permita proponer estrategias que guíen a los diferentes actores sociales, económicos y políticos hacia prácticas comprometidas con el cumplimiento de los derechos de las personas con capacidad intelectual límite, en coherencia con la Convención sobre los Derechos de las Personas con Discapacidad ratificada por España en 2008. En este artículo, se presentan los resultados principales del mencionado estudio.

Aunque encontramos en diversas investigaciones diversas referencias conceptuales sobre lo que conocemos como personas con capacidad intelectual límite, resultan en general escasos en el conocimiento sobre su situación social, demográfica y personal actual, así como en la medida en que la sociedad reconoce a las personas con capacidad límite como sujetos de derechos. Es por ello que esta investigación conllevó como objetivos:

- Identificar aspectos que facilitan/dificultan la inclusión social de las personas con capacidad intelectual límite.

- Conocer el uso y satisfacción con los recursos de apoyo disponibles para una inclusión social efectiva.

- Identificar experiencias exitosas y buenas prácticas para avanzar y retroalimentar el quehacer de los diferentes servicios públicos y privados en pro de las personas con capacidad intelectual limite.
- Realizar propuestas de mejora para la prestación de apoyos según las necesidades detectadas.

\section{Metodología}

El alcance de estos objetivos ha sido posible mediante una estrategia metodológica triangular (Denzin, I989), integrando un trabajo de revisión bibliográfica con una perspectiva cuantitativa de los datos y una perspectiva cualitativa sobre las vivencias y experiencias de las personas con capacidad intelectual límite, familiares y profesionales, primando el discurso propio de las personas y las familias, cuyas aportaciones son fundamentales para orientar un enfoque o modelo de intervención biopsicosocial pertinente.

En una primer fase de la investigación se obtuvo una aproximación cuantitativa sociodemográfica de la población con capacidad intelectual límite a través de la revisión y explotación de fuentes estadísticas, validadas oficialmente, como la Base de Datos Estatal de Discapacidad (BDEPD, 20I2) y la Encuesta de Discapacidad, Autonomía Personal y Situaciones de Dependencia (EDAD, 2008). Paralelamente, se abordó el marco teórico-científico existente, que significó la sistematización del conocimiento prevalente sobre las personas con capacidad intelectual límite a través de una gestión documental y de información procedente de fuentes oficiales científicas y divulgativas, que aglutinaban estudios e investigaciones sobre el objeto de análisis. El proceso de análisis de contenido permitió definir de forma más precisa las líneas de investigación, el marco teóricoconceptual y el enfoque metodológico para la construcción y aplicación de las técnicas de investigación cualitativa para obtener los datos primarios.

En una segunda fase, atendiendo al alcance descriptivo de la investigación y, por otro lado, a la invisibilidad social de las personas con 
capacidad intelectual límite, como ya se ha señalado, fue decisiva una estrategia de recogida de datos primarios eminentemente cualitativa, con mayor énfasis en las vivencias y experiencias prácticas de las propias personas con capacidad intelectual límite y sus familias. El diseño conllevó la definición de perfiles diferentes, que respondieran a las unidades de estudio y el tipo de relación, personas con capacidad intelectual límite, familias, profesionales y expertos. Los criterios de selección incluyeron variables diferentes y complementarias relativas a las dimensiones de los constructos de inclusión social y calidad de vida. En el caso de las personas con capacidad intelectual límite y familias, se contemplaron variables como la edad, el género y la ubicación territorial. En relación con los profesionales se tuvo en cuenta el tipo de profesión, entidad de pertenencia y apoyo o atención que prestaban. Por último, los perfiles de los expertos fueron seleccionados por su relación con el ámbito de la investigación y discapacidad intelectual. De acuerdo a los criterios anteriores de la población objeto de estudio, se realizaron nueve entrevistas en profundidad tal como se detalla en la Tabla I.

Atendiendo al criterio de validación de la información, aportada desde el discurso social individual y colectivo, fue complementaria la recogida de datos primarios a partir de la aplicación de un cuestionario online, con preguntas abiertas y cerradas referidas a las variables del estudio, a una muestra de 93 profesionales de todas las entidades portadoras de servicios para las personas con capacidad intelectual límite a nivel estatal y autonómico.

Sus aportaciones fueron de gran utilidad para describir las principales barreras del entorno social que limitan la participación activa de las personas con capacidad intelectual límite y, por otro lado, de aquellos factores de protección que favorecen la inclusión social, mostrando un conjunto de propuestas y prácticas de apoyo más coherentes con las necesidades de estas personas y sus familias.

Los criterios de análisis seguidos en la última fase de generación de conocimiento objetivo fueron: la horizontalidad temática, la referencia a factores de riesgo y factores de protección ante la exclusión social, la referencia a propuestas de actuación para la inclusión de las personas con capacidad intelectual límite y la perspectiva de los perfiles entrevistados (persona, familiar, profesional). Otras variables tenidas en cuenta de forma transversal fueron la etapa del ciclo vital, el diagnóstico, el grado de autonomía personal y el rol social y profesional, principalmente.

Tabla 1. Perfiles de entrevistas realizadas
\begin{tabular}{|l|}
\hline Perfil \\
\hline $\begin{array}{l}\text { 1. Mujer con capacidad intelectual límite, entre } 30 \text { y } 35 \text { años, adulta en situación de inclusión social } \\
\text { normalizada y vida independiente plena }\end{array}$ \\
\hline $\begin{array}{l}\text { 2. Varón con capacidad intelectual límite, entre } 30 \text { y } 35 \text { años, adulta en situación de inclusión social } \\
\text { normalizada, pero dependiente de familiares }\end{array}$ \\
\hline $\begin{array}{l}\text { 3. Varón con capacidad intelectual límite, entre } 30 \text { y } 35 \text { años, dependiente de la familia y autonomía personal } \\
\text { y social en proceso }\end{array}$ \\
\hline 4. Mujer, directiva de asociación de apoyo a personas con capacidad intelectual límite \\
\hline 5. Madre con hijo joven con capacidad intelectual límite \\
\hline 6. Madre con hija joven con capacidad intelectual límite \\
\hline 7. Mujer técnica de una asociación, responsable del área de deporte \\
\hline $\begin{array}{l}\text { 8. Mujer técnica responsable de área de autonomía personal y social, hermana de persona con capacidad } \\
\text { intelectual límite }\end{array}$ \\
\hline 9. Experto en investigación y personas con discapacidad intelectual \\
\hline
\end{tabular}

Fuente: elaboración propia. 


\section{Resultados}

\subsection{Investigación actual, realidad social y personas con capacidad intelectual límite}

La producción investigadora y científica, marco de referencia teórico del estudio, sobre las personas con capacidad intelectual límite ha mostrado que existe, en general, escaso reconocimiento, social y político, de sus derechos y necesidades. Los principales avances en el reconocimiento formal de las personas con capacidad intelectual límite han sido promovidos por las entidades sociales, con énfasis en la necesidad de una acotación definitoria precisa de la capacidad intelectual límite, más allá del cociente intelectual (Palos, 2002).

Según Artigas Pallarés (20II) los individuos con capacidad de inteligencia límite (CIL) presentan unas limitaciones escolares, sociales y laborales similares a las del retraso mental (RM), aunque de menor grado. La condición que define la CIL es la detección de un cociente intelectual comprendido entre 7I y 84 . A diferencia del RM, el colectivo de pacientes con CIL ha merecido poca atención en las publicaciones médicas, por lo que están poco definidas las características cognitivas de este grupo.

Las entidades representativas, familias y personas con capacidad intelectual límite acogen con mayor frecuencia la referencia a personas que, con un grado leve de discapacidad intelectual, pueden alcanzar una calidad de vida y un nivel de autonomía y autogobierno estándares, mediante apoyos puntuales y respecto a algunas esferas vitales (VVAA, 2004).

Los modelos y estrategias metodológicas actuales para la intervención con personas con capacidad intelectual límite se centran, principalmente, en la importancia de los apoyos, como factor clave para que puedan interactuar inclusivamente en la comunidad, contexto relevante para su experiencia vital. Uno de dichos modelos más constantemente referenciados es el enfoque de calidad de vida y los apoyos, precisamente por hacer énfasis en la individualidad de las personas. Este enfoque es el que mejor abordaría la promoción de una vida independiente de las personas con capacidad intelectual e inclusión social efectiva. Al mismo tiempo, se hace hincapié en el enfoque de los derechos de las personas con discapacidad ${ }^{2}$, con especial interés en el principio de igualdad, expresado en la importancia de respetar la perspectiva de género.

Siguiendo estos enfoques, desde la perspectiva de los hitos clave del ciclo vital de las personas con capacidad intelectual límite y las oportunidades reales que le ofrece el entorno social para su desarrollo personal y competencial, es esta realidad la que aporta más información para entender sus necesidades y las actuaciones que han de prevalecer para impulsar la inclusión social efectiva. Un dominio clave es en el ámbito educativo, en el que los protagonistas del estudio, familias y profesionales, resaltan la importancia de la adaptación curricular a las necesidades educativas específicas de las personas con capacidad intelectual límite, la sensibilización de los iguales, la preparación de los profesionales y la continuidad en el sistema educativo o la formación continua, con especial énfasis en la formación ocupacional, así como la transición de la educación al mundo laboral (Giné 2006).

El ámbito laboral, precisamente, es otro de los ámbitos sociales más identificados como excluyentes para las personas con capacidad intelectual límite. Se enfatiza la necesaria revisión aquellos enfoques de intervención sociolaboral, centrados en lugares cerrados e institucionalizados, hacia otras estrategias de ubicación de las personas con capacidad intelectual límite en el entorno ordinario, optando por los mismos recursos disponibles para la ciudadanía en general, mostrándose como modelo eficaz el empleo con apoyo (Egido et al., 2009).

2. Convención de Naciones Unidas sobre los Derechos Humanos de las personas con discapacidad, ratificada por España en 2008 y trasladada al marco legal actual. 
Otra área temática con abundante referencia bibliográfica es la autonomía personal y la vida independiente, cuyo principal exponente es el ocio inclusivo al representar una alternativa viable para el aprendizaje de una vida autónoma. Además, el recurso del ocio y tiempo libre favorece la toma de sus propias decisiones y la relación con los iguales.

Otros temas referenciados en el análisis documental, también importantes, son el rol de las familias, el soporte que implica para las personas con capacidad intelectual límite y la actitud de las mismas. Son considerados los apoyos naturales por excelencia, junto con los hermanos, compañeros de estudios y de trabajo (Riera et al., 2003).

Hay ámbitos de estudio con mayor profundización que otros, lo cual es indicativo de posibles fortalezas y vacíos en el ámbito de la investigación científica y divulgativa, imprescindibles de acometer, que respondan a las principales demandas y necesidades de las personas con capacidad intelectual límite y sus familias. Ejemplo de la necesidad de una mayor investigación es el ámbito del acceso al empleo, como ya hemos señalado, la formación profesional, el acceso a los servicios y recursos en las zonas rurales o la relación entre el sistema de la dependencia y las personas con capacidad intelectual límite. También, de acuerdo al modelo social de la discapacidad, corresponde avanzar en el estudio de los apoyos y factores facilitadores de una mayor autonomía personal y vida independiente, en favor del derecho de autodeterminación. Frontera y Gómez Bahillo (2013) muestran esta realidad desde la óptica de la práctica diaria que se desarrolla en las organizaciones representativas y desde la visión de las personas con capacidad intelectual límite y las familias.

\subsection{La realidad sociodemográfica de los datos}

Desde la mirada cuantitativa de los datos se abordaron, como primer criterio metodológico de estimación, las fuentes oficiales estadísticas más fiables, base para acercarnos de forma más precisa a la realidad sociodemográfica de las personas con discapacidad. El segundo criterio fue definir como población objetivo a aquellas personas que, además de un antecedente médico o diagnóstico sobre su capacidad intelectual límite, han accedido a algún tipo de reconocimiento oficial de la discapacidad (subjetiva u objetiva).

A partir de las diferentes fuentes oficiales utilizadas, se ha procedido a realizar una estimación de la población con discapacidad a partir de diferentes criterios médicos y funcionales que las fuentes disponibles proveen:

- Población mínima registrada: personas que han sido reconocidas oficialmente con discapacidad, específicamente por capacidad intelectual límite y tienen un grado inferior al $33 \%$.

- Diagnósticos relacionados registrados: personas que han sido reconocidas con discapacidad, específicamente por algún tipo de deficiencia intelectual (excluyendo demencias) y tienen un grado inferior al $33 \%$.

- Población mínima estimada: personas que han reconocido dificultades en áreas prevalentes para las personas con capacidad intelectual límite, por causa de inteligencia límite, con cualquier severidad.

- Total población registrada: personas que han sido reconocidas con discapacidad específicamente por inteligencia límite o diagnósticos relacionados y tienen un grado inferior al $33 \%$.

- Diagnósticos relacionados estimados: personas que han reconocido dificultades en áreas prevalentes para las personas con capacidad intelectual límite, por causa de alguna deficiencia intelectual (excluyendo demencias), con severidad no grave.

- Total población estimada: personas que han reconocido dificultades en áreas prevalentes para las personas con capacidad intelectual límite, por causa de inteligencia límite o alguna deficiencia intelectual (excluyendo demencias), con severidad no grave. 
Tabla 2. Población con capacidad intelectual límite en España, por sexo, según diferentes hipótesis estimativas. Total personas

\begin{tabular}{|c|c|c|c|}
\hline Hipótesis & Mujeres & Varones & Total \\
\hline Población mínima registrada & 2.528 & 4.330 & 6.858 \\
\hline Diagnósticos relacionados (registrada) & 3.726 & 6.953 & 10.679 \\
\hline Población mínima (estimada) & 5.120 & 6.499 & 11.619 \\
\hline Total población (registrada) & 6.254 & 11.283 & 17.537 \\
\hline Diagnósticos relacionados (estimada) & 18.834 & 29.741 & 48.575 \\
\hline Total población (estimada) & 23.954 & 36.240 & 60.194 \\
\hline
\end{tabular}

Fuente: elaboración propia a partir de la Base de Datos Estatal de Discapacidad (BDEPD, 20I 2 ) y de la de la encuesta de Discapacidad, Autonomía personal y situaciones de Dependencia (EDAD, 2008).

Tabla 3. Población con inteligencia límite por grupo de edad y sexo. Total y porcentaje

\begin{tabular}{|c|c|c|c|c|c|c|}
\hline & \multicolumn{3}{|c|}{ Población estimada } & \multicolumn{3}{|c|}{ Población registrada } \\
\hline & Varones & Mujeres & Total & Varones & Mujeres & Total \\
\hline De 0 a 16 & 12.627 & 3.626 & 16.253 & 3.912 & 2.106 & 6.018 \\
\hline De 17 a 33 & 7.700 & 4.961 & 12.661 & 3.661 & 1.780 & 5.441 \\
\hline De 34 a 50 & 8.893 & 4.243 & 13.136 & 2.669 & 1.504 & 4.173 \\
\hline De 51 a 67 & 4.615 & 4.908 & 9.523 & 818 & 560 & 1.378 \\
\hline De 68 a 84 & 2.217 & 4.843 & 7.060 & 192 & 263 & 455 \\
\hline De 85 o más & 187 & 1.373 & 1.560 & 24 & 36 & 60 \\
\hline Dotal & 36.239 & 23.954 & 60.193 & 11.276 & 6.249 & 17.525 \\
\hline
\end{tabular}

Fuente: elaboración propia a partir de la Base de Datos Estatal de Discapacidad (BDEPD, 20I2) y de la de la encuesta de Discapacidad, Autonomía personal y situaciones de Dependencia (EDAD, 2008).

Tomando como referencia la clasificación anterior, es posible afirmar que al menos 60.I94 personas en España disponen de un diagnóstico de inteligencia límite o que, en todo caso, encuentran dificultades de tipo moderado para realizar tareas de la vida diaria, por causa relacionada con una capacidad intelectual límite. En términos demográficos, se trata de una población joven, con un patrón de género de aproximadamente tres varones por cada dos mujeres.

Como podemos observar, la población con capacidad intelectual límite es una población mayoritariamente invisible a los servicios de valoración de discapacidad, especialmente en el caso de las mujeres. De hecho, se evidencian diferencias significativas por sexo en lo que respecta a población estimada y población registrada.

Según su distribución por comunidades autónomas, podemos observar en la tabla siguiente el porcentaje mayor de personas con capacidad intelectual límite localizadas en Andalucía (I7\%), seguida de Madrid (I 6,4\%), Comunidad Valenciana ( $5 \%$ ) y Cataluña ( $13 \%$ ).

Por nivel de estudios, se perfilan tres grupos: sin estudios $(34,6 \%)$, con estudios primarios $(35,4 \%)$ y con secundarios o superiores $(3 \mathrm{I}, 7 \%)$. Las mujeres presentan un nivel de estudios inferior al de los varones, de manera sistemática. 
Gráfico 1. Población con capacidad intelectual límite en España por sexo, según diferentes hipótesis estimativas. Porcentaje
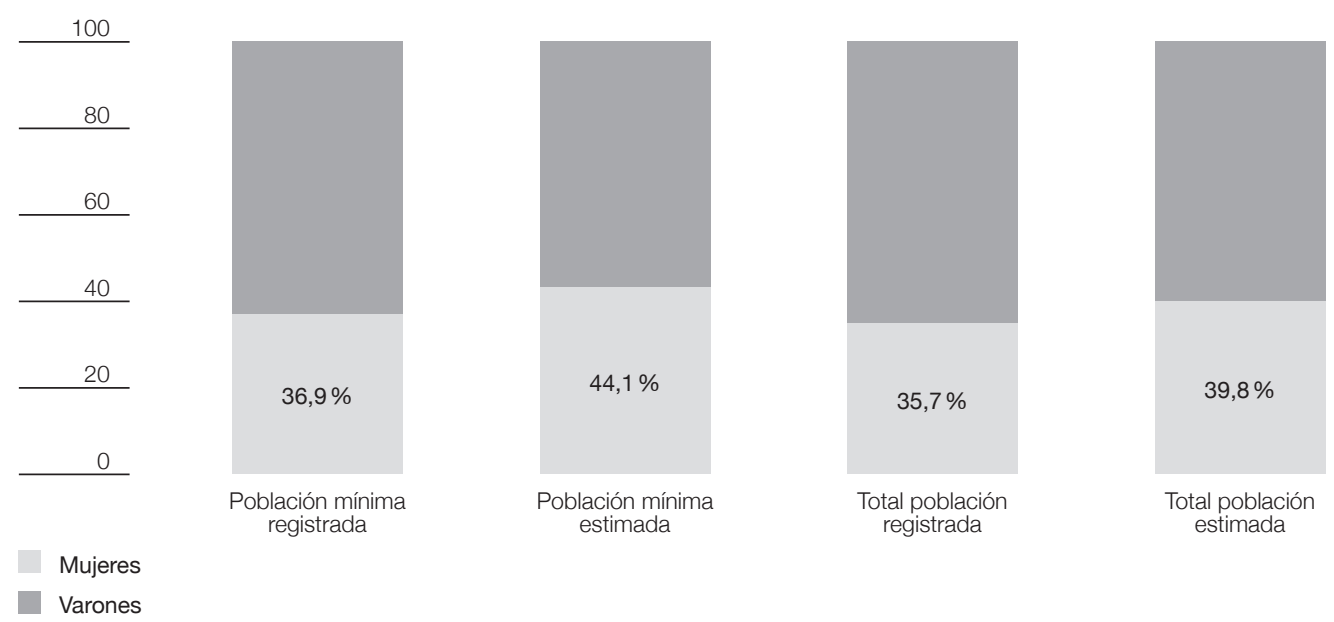

Fuente: elaboración propia a partir de la Base de Datos Estatal de Discapacidad (BDEPD, 20I2) y de la de la encuesta de Discapacidad, Autonomía personal y situaciones de Dependencia (EDAD, 2008).

\section{Tabla 4. Población con inteligencia límite por CCAA de residencia. Total personas}

\begin{tabular}{|l|c|}
\cline { 2 - 2 } \multicolumn{1}{l|}{} & Frecuencia \\
\hline Andalucía & 10.192 \\
\hline Aragón & 1.049 \\
\hline Principado de Asturias & 804 \\
\hline Illes Balears & 1.422 \\
\hline Canarias & 2.562 \\
\hline Cantabria & 804 \\
\hline Castilla y León & 3.569 \\
\hline Castilla-La Mancha & 1.723 \\
\hline Cataluña & 7.802 \\
\hline Comunidad Valenciana & 9.212 \\
\hline Extremadura & 2.891 \\
\hline Galicia & 3.900 \\
\hline Comunidad de Madrid & 9.875 \\
\hline Región de Murcia & 1.889 \\
\hline Comunidad Foral de Navarra & 208 \\
\hline País Vasco & 2.054 \\
\hline La Rioja & 135 \\
\hline Ceuta y Melilla & 103 \\
\hline Total & 60.194 \\
\hline
\end{tabular}

Fuente: elaboración propia a partir de la encuesta de Discapacidad, Autonomía personal y situaciones de Dependencia (EDAD, 2008). Las celdas con cifras inferiores a 5.000 personas pueden estar afectadas de errores de muestreo. 
Tabla 5. Población con inteligencia límite según nivel de estudios terminados. Total y porcentaje

\begin{tabular}{|l|c|c|c|c|c|}
\cline { 2 - 5 } \multicolumn{1}{c|}{} & Varón & Mujer & Total & Porcentaje & $\%$ Mujeres \\
\hline No sabe leer ni escribir & 1.212 & 1.425 & 2.637 & $6,0 \%$ & $7,0 \%$ \\
\hline Estudios primarios incompletos & 4.771 & 5.616 & 10.387 & $23,6 \%$ & $27,6 \%$ \\
\hline Estudios primarios o equivalentes & 9.445 & 7.188 & 16.633 & $37,9 \%$ & $35,4 \%$ \\
\hline Estudios secundarios o superiores & 9.274 & 6.442 & 15.716 & $35,8 \%$ & $31,7 \%$ \\
\hline
\end{tabular}

Fuente: elaboración propia a partir de la encuesta de Discapacidad, Autonomía personal y situaciones de Dependencia (EDAD, 2008). Las celdas con cifras inferiores a 5.000 personas pueden estar afectadas de errores de muestreo.

Tabla 6. Población con inteligencia límite en edad escolar según situación respecto a la inclusión
educativa. Total y porcentaje
\begin{tabular}{|l|c|c|c|c|c|}
\hline No escolarizado & Varón & Mujer & Total & Porcentaje & $\%$ Mujeres \\
\hline $\begin{array}{l}\text { Escolarizado en un centro o aula de educación } \\
\text { especial }\end{array}$ & 1.649 & 104 & 1.753 & $10,8 \%$ & $2,9 \%$ \\
\hline $\begin{array}{l}\text { Escolarizado en un centro ordinario en régimen } \\
\text { de integración y recibiendo apoyos especiales }\end{array}$ & 5.811 & 2.411 & 8.222 & $50,6 \%$ & $66,5 \%$ \\
\hline $\begin{array}{l}\text { Escolarizado en un centro ordinario sin ningún } \\
\text { tipo personalizado de apoyo }\end{array}$ & 3.656 & 333 & 3.989 & $24,5 \%$ & $9,2 \%$ \\
\hline NC & 422 & 434 & 856 & $5,3 \%$ & $12,0 \%$ \\
\hline
\end{tabular}

Fuente: elaboración propia a partir de la encuesta de Discapacidad, Autonomía personal y situaciones de Dependencia (EDAD, 2008). Las celdas con cifras inferiores a 5.000 personas pueden estar afectadas de errores de muestreo.

\begin{tabular}{|c|c|c|c|c|c|}
\hline & Varón & Mujer & Total & Porcentaje & $\%$ Mujeres \\
\hline Trabajando & 5.098 & 2.205 & 7.303 & $16,6 \%$ & $10,8 \%$ \\
\hline En desempleo & 1.988 & 1.252 & 3.240 & $7,4 \%$ & $6,2 \%$ \\
\hline Percibiendo pensión & 12.241 & 8.503 & 20.744 & $47,2 \%$ & $41,8 \%$ \\
\hline Otra situación & 5.375 & 8.711 & 14.086 & $32,1 \%$ & $42,9 \%$ \\
\hline
\end{tabular}

Fuente: elaboración propia a partir de la encuesta de Discapacidad, Autonomía personal y situaciones de Dependencia (EDAD, 2008). Las celdas con cifras inferiores a 5.000 personas pueden estar afectadas de errores de muestreo.

En lo relacionado con la inclusión educativa, aproximadamente dos terceras partes de la población en edad escolar se encuentra escolarizada en centro ordinario (con o sin apoyos). En este caso las mujeres presentan mayor nivel de inclusión educativa.
La situación sociolaboral de las personas con capacidad intelectual límite es precaria, tal como refleja la información disponible sobre la participación en el mercado de trabajo: menos del $17 \%$ se encuentra trabajando y casi la mitad se encuentra percibiendo una pensión. 
En cuanto a su percepción subjetiva de salud, de aquellos que respondieron a la encuesta, (60.193) más del 60\% de la población $(90 \%$ en el caso de las mujeres), reconocen tener un problema de salud crónico. Sólo un 7,9\% señala que posee una salud muy buena.

Respecto a su entorno social, prevalecen aquellas personas con capacidad intelectual límite que residen en el domicilio de sus padres. Una cuarta parte reconoce tener dificultades para relacionarse con amistades o personas cercanas y dedican su tiempo libre, fundamentalmente, al consumo de televisión. Utilizan el transporte público de forma mayoritaria. La movilidad es baja y su hábitat social siempre está muy cerca de sus padres.

En general, la población con capacidad intelectual límite reconoce no haber sentido rechazo o discriminación por discapacidad $(67,5 \%)$, o sólo algunas veces $(2 \mathrm{I}, 3 \%)$. Aproximadamente una de cada cinco personas reconoce pertenecer a una ONG por causa de discapacidad.

\begin{tabular}{|c|c|c|c|c|c|}
\hline & Varón & Mujer & Total & Porcentaje & $\%$ Mujeres \\
\hline Muy buena & 3.771 & 999 & 4.770 & $7,9 \%$ & $4,2 \%$ \\
\hline Buena & 18.424 & 10.363 & 28.787 & $47,8 \%$ & $43,3 \%$ \\
\hline Regular & 10.364 & 9.157 & 19.521 & $32,4 \%$ & $38,2 \%$ \\
\hline Mala & 3.500 & 2.382 & 5.882 & $9,8 \%$ & $9,9 \%$ \\
\hline Muy mala & 181 & 1.054 & 1.235 & $2,1 \%$ & $4,4 \%$ \\
\hline NC & 0 & 0 & 0 & $0,0 \%$ & $0,0 \%$ \\
\hline
\end{tabular}

Fuente: elaboración propia a partir de la encuesta de Discapacidad, Autonomía personal y situaciones de Dependencia (EDAD, 2008). Las celdas con cifras inferiores a 5.000 personas pueden estar afectadas de errores de muestreo.

Tabla 9. Población con inteligencia límite según lugar de residencia respecto a la residencia de sus padres. Total y porcentaje

\begin{tabular}{|l|c|c|c|c|c|}
\cline { 2 - 6 } \multicolumn{1}{c|}{} & Varón & Mujer & Total & Porcentaje & $\%$ Mujeres \\
\hline En el mismo domicilio & 24.291 & 10.632 & 34.923 & $58,0 \%$ & $44,4 \%$ \\
\hline En el mismo edificio & 371 & 0 & 371 & $0,6 \%$ & $0,0 \%$ \\
\hline En el mismo barrio o pueblo & 1.131 & 1.375 & 2.506 & $4,2 \%$ & $5,7 \%$ \\
\hline En la misma ciudad & 1.514 & 0 & 1.514 & $2,5 \%$ & $0,0 \%$ \\
\hline En la misma provincia & 1.667 & 250 & 1.917 & $3,2 \%$ & $1,0 \%$ \\
\hline En distinta provincia & 441 & 519 & 960 & $1,6 \%$ & $2,2 \%$ \\
\hline En otro país & 0 & 139 & 139 & $0,2 \%$ & $0,6 \%$ \\
\hline No tiene (padres) & 6.344 & 10.316 & 16.660 & $27,7 \%$ & $43,1 \%$ \\
\hline No sabe & 0 & 0 & 0 & $0,0 \%$ & $0,0 \%$ \\
\hline NC & 480 & 724 & 1.204 & $2,0 \%$ & $3,0 \%$ \\
\hline
\end{tabular}

Fuente: elaboración propia a partir de la encuesta de Discapacidad, Autonomía personal y situaciones de Dependencia (EDAD, 2008). Las celdas con cifras inferiores a 5.000 personas pueden estar afectadas de errores de muestreo. 
Tabla 10. Población con inteligencia límite que se ha sentido discriminada por motivo de discapacidad en los últimos 12 meses. Total y porcentaje

\begin{tabular}{|l|c|c|c|c|c|}
\cline { 2 - 5 } \multicolumn{1}{c|}{} & Varón & Mujer & Total & Porcentaje & $\%$ Mujeres \\
\hline Nunca & 23.080 & 17.577 & 40.657 & $67,5 \%$ & $96,4 \%$ \\
\hline Algunas veces & 9.068 & 3.748 & 12.816 & $21,3 \%$ & $37,9 \%$ \\
\hline Muchas veces & 2.358 & 1.461 & 3.819 & $6,3 \%$ & $9,8 \%$ \\
\hline Constantemente & 1.244 & 333 & 1.577 & $2,6 \%$ & $5,2 \%$ \\
\hline NC & 490 & 836 & 1.326 & $2,2 \%$ & $2,0 \%$ \\
\hline
\end{tabular}

Fuente: elaboración propia a partir de la encuesta de Discapacidad, Autonomía personal y situaciones de Dependencia (EDAD, 2008). Las celdas con cifras inferiores a 5.000 personas pueden estar afectadas de errores de muestreo.

Tabla 11. Población con inteligencia límite que pertenece a una ONG por causa de discapacidad. Total y porcentaje

\begin{tabular}{|l|c|c|c|c|c|}
\cline { 2 - 5 } \multicolumn{1}{c|}{} & Varón & Mujer & Total & Porcentaje & $\%$ Mujeres \\
\hline Pertenece a alguna ONG & 4.855 & 3.876 & 8.731 & $19,9 \%$ & $19,1 \%$ \\
\hline
\end{tabular}

Fuente: elaboración propia a partir de la encuesta de Discapacidad, Autonomía personal y situaciones de Dependencia (EDAD, 2008). Las celdas con cifras inferiores a 5.000 personas pueden estar afectadas de errores de muestreo.

\subsection{Necesidades y demandas de las personas con capacidad intelectual límite}

Hasta aquí se ha descrito una imagen aproximada de la situación sociodemográfica de las personas con inteligencia límite, mostrando los datos fundamentales relacionados con la inclusión social, como es el acceso a la educación, al empleo y al medio social (Laparra, 2007), como principales ámbitos que limitan la inclusión efectiva en la sociedad y, por ende, la oportunidad de llevar una vida autónoma e independiente.

Desde la perspectiva más subjetiva, extraída del análisis cualitativo del discurso, desde lo vivencial de las personas y las familias, y desde el punto de vista de las entidades y profesionales que gestionan los servicios y recursos disponibles en la actualidad, emerge un discurso profundo sobre cuáles son las principales necesidades y demandas de las personas con capacidad intelectual límite, que requieren una respuesta eficaz desde los diferentes ámbitos de la sociedad, siendo clave la voz activa y el derecho de autodeterminación de los propios protagonistas.

El discurso de las personas con capacidad intelectual límite y las familias expresa un diagnóstico de la realidad que se repite y se consolida desde ambas perspectivas: no reconocimiento de la señales más frecuentes de las limitaciones funcionales asociadas a la capacidad intelectual límite hasta comenzar los primeros síntomas en el ámbito educativo (principalmente en secundaria), a medida que es más exigente el medio, donde el desajuste cognitivo se muestra con más claridad, además de tener dificultades en habilidades sociales. Es la etapa en la que comienza a haber una mayor diferenciación de intereses y expectativas con sus grupos de iguales. La dificultad de un diagnóstico a tiempo y, por tanto, del acceso a los servicios de Atención Temprana, retrasa la disponibilidad de los apoyos oportunos en cada etapa del ciclo vital para potenciar su desarrollo personal y social posterior. Ello 
confirma a Artigas (2007): "En la mayoría de los casos las dificultades de estos niños no han sido detectadas en el colegio porque pasan desapercibidos -apunta Artigas-. Lo único que se sabe es que no funcionan al mismo ritmo que los demás y el error más generalizado es pensar que son vagos, que están desmotivados o que la culpa es de los padres porque no se preocupan lo suficiente".

Normalmente, ante estas dificultades las familias acuden a las entidades asociativas o profesionales, de forma privada, para obtener un diagnóstico que les permita entender cuál es el tipo de discapacidad y características de sus familiares. Surgen, entonces, determinados apoyos específicos y especializados, tales como psicólogos, logopedas, apoyos educativos particulares, etc. No obstante, es importante señalar los avances significativos en la Atención Temprana de las personas con capacidad intelectual límite desde un tiempo atrás hasta la actualidad.

La necesidad de disponer de un certificado de discapacidad se hace aún más relevante para abordar los diferentes apoyos que necesitan y acceder a determinadas ayudas, que son claves para recuperar capacidades o para aminorar las consecuencias de una atención deficitaria anterior.

Al mismo tiempo, las familias acuden cada vez con mayor frecuencia a entidades sociales que ofertan una serie de servicios y recursos, a veces generales para todas las personas con discapacidad, y otras (de forma minoritaria) específicas. Van de un centro a otro, en la búsqueda del apoyo más ajustado a la necesidad de la persona, mientras la misma persona va tomando conciencia de sus limitaciones funcionales, que en muchos casos no asumen hasta la misma adolescencia.

Respecto al ámbito educativo, señalan situaciones de continuidad del proceso formativo en la educación normalizada, en el que encontraban, por un lado, profesionales que limitaban su aprendizaje y, por otro lado, profesionales que les guiaban hacia una formación adaptada a sus características. El paso de la etapa educativa a la laboral conlleva dificultades, precisamente, por la insuficiente formación ocupacional a la que tienen disponibilidad. Siguen siendo las entidades sociales el principal recurso para dar respuesta a esta situación, ante la falta de opciones de formación profesional del sector público. Esto les conduce a una frustración permanente a la hora de acceder al mercado de trabajo.

La dificultad de un trabajo coordinado entre todos los actores relacionados con el mercado de trabajo es otra variante influyente. Se añade, además, la ambigüedad y vacío del marco legal respecto a la inclusión sociolaboral de las personas con capacidad intelectual límite. Además, el entorno social no está adaptado para que las personas con capacidad intelectual límite puedan relacionarse con otros iguales, sufriendo en muchos casos el aislamiento social. Son las opciones de ocio y tiempo libre ofertadas por las propias entidades representativas las que les abren una oportunidad de aprendizaje en una dimensión fundamental para estas personas como es la toma de decisiones, potenciando al mismo tiempo la capacidad de autodeterminación.

En cuanto al discurso de los padres, las expectativas que muestran sus hijas e hijos no responden a la realidad de su situación de discapacidad. Desean asumir roles de adultos, aunque señalan el sentimiento de frustración en el logro deseado de una vida independiente. Hay un sentimiento de soledad durante un largo período de tiempo por no haber una comprensión por parte del entorno escolar y social de la capacidad intelectual límite, negándoseles el acceso a aquellos apoyos que hubieran evitado que entraran en una dinámica de abusos y posibles conductas que conllevan riesgos sociales en muchos casos, como reconocen las mismas personas con capacidad intelectual límite.

Los apoyos naturales son importantes, pero al no tener las personas con capacidad intelectual límite conciencia clara de sus limitaciones 
funcionales se pueden convertir en apoyos insuficientes para que puedan optar a una vida independiente.

Son precisamente los hermanos los apoyos naturales fundamentales en su etapa de transición a la vida adulta (Pallisera et al., 2013). Aunque, como señalan estos, al mismo tiempo, en determinadas situaciones se generan tensiones familiares por la propia dinámica familiar que requiere de una mayor atención a la persona con inteligencia límite. Es un apoyo natural que se hace aún más importante a medida que pasa el tiempo por la desaparición de los padres, situación de estrés familiar frecuente. La constitución de redes de apoyos familiares es clave para favorecer la inclusión.

En el relato de las familias y las personas con capacidad intelectual límite se observa también la perspectiva de género respecto a las consecuencias finales para las personas. En el caso de la mujer, sus derechos son más vulnerados. La invisibilidad de la propia discapacidad, el desfase cognitivo o el contexto cultural tradicional del rol de la mujer favorecen posibles abusos o situaciones de discriminación. Las familias comentan con mucha preocupación la exposición de las personas con capacidad intelectual límite a riesgos de abusos sociales, como por ejemplo riesgos de drogadicción y de conductas delictivas, sin que ellas mismas sean conscientes del impacto negativo.

De esta forma, los principales factores de riesgo que señalan las personas con capacidad intelectual límite y las familias son:

- La situación socioeconómica familiar junto a las escasas opciones de ayudas, que dificultan disponer de apoyos pertinentes en su preparación para la vida adulta y vida independiente.

- Insuficiente preparación de los profesionales en el ámbito educativo para prestar apoyos específicos desde la perspectiva de las personas con capacidad intelectual límite.

- La ausencia de pertenencia o de identificación con el grupo natural positivo de referencia. Este riesgo en la adolescencia, en aquellas personas que no son conscientes de su discapacidad o que se ubican en un entorno de riesgo, les hace más vulnerables a la hora de entrar en la dinámica de conductas disruptivas y complejas.

- Desorientación permanente del sistema respecto a las necesidades y demandas de las personas con capacidad intelectual límite y las familias.

Y como factores de protección, las familias y las personas con capacidad intelectual límite mencionan:

- Disponibilidad de apoyos en la administración y en las entidades sociales, que puedan ser accesibles con ayudas ante la dificultad económica de muchas familias (especialmente en las zonas rurales), que eviten el aislamiento de las personas con capacidad intelectual límite.

- Una mayor sensibilización de la sociedad respecto de la realidad de las personas con capacidad intelectual límite.

- El abordaje de un modelo diferente desde las asociaciones ante los avances significativos en el conocimiento de las personas con capacidad intelectual límite y sus necesidades de apoyo.

La otra mirada del discurso, relevante para entender las necesidades de las personas con capacidad intelectual límite, son los gestores de las entidades sociales y los profesionales que participan de las mismas. En este sentido, el aprendizaje de los gestores de las entidades representativas, coincide en gran parte con la visión de las personas con capacidad intelectual límite y sus familias:

- Aunque es importante un buen diagnóstico, señalan que hay que tener cuidado con la percepción social que conlleva estar etiquetado dentro de un colectivo.

- Confirman que las mayores dificultades se dan en la etapa de secundaria, observándose las diferencias con sus 
iguales y la repercusión del correspondiente aislamiento. Por otro lado, las personas en el sistema educativo pasan desapercibidas, y las conductas disruptivas no se asocian a dificultades cognitivas u otras.

- Redundan en el sentido inclusivo del empleo para las personas con capacidad intelectual límite. La escasez de programas de cualificación y la no adaptación de la formación profesional para la obtención de un empleo implican, para las personas con capacidad intelectual límite, la insuficiente cualificación para acceder a un puesto de trabajo en el mercado ordinario.

- Se vuelve a enfatizar al vacío existente en la coordinación de todos los actores relacionados con el mercado de trabajo. Además, apuntan que la legislación no visibiliza lo suficiente a las personas con capacidad intelectual límite. Por ello, la exigencia legal de ser reconocido con un $33 \%$ para que sean sujetos de derecho en las cuotas de contratación obligatorias deja fuera a muchas personas con capacidad intelectual límite. Llegan a ser las personas con discapacidad más vulnerables del sistema, en este sentido.

- El contexto familiar sigue siendo importante para favorecer las relaciones sociales y su inclusión social, así como los grupos de amigos. Estos últimos, más los apoyos naturales son claves para prevenir determinadas conductas delictivas, ante a vulnerabilidad que implica la necesidad de pertenecer a un grupo social.

- Es importante considerarlos con capacidad suficiente para ejercer los derechos que le corresponden constitucionalmente a la ciudadanía, como el derecho a votar. Antes de llegar a la figura legal de incapacitación debe formarse el núcleo familiar principal. Debe predominar el derecho a la autonomía personal y social.

- En línea con lo anterior, el ocio inclusivo es fundamental como instrumento para favorecer las relaciones sociales, las habilidades sociales, la autonomía personal y la toma de decisiones. Deben compartir los espacios sociales normales pero con grupos de iguales, generando sus propias pandillas naturales.

- Las instituciones responsables de proteger los derechos y la convivencia social no están suficientemente sensibilizadas, encontrándose situaciones de personas reclusas con capacidad intelectual límite que no han podido tener la defensa correspondiente adaptada o son muy vulnerables en el ámbito penitenciario.

Desde la percepción de los profesionales, que representan apoyos imprescindibles para el desarrollo personal y social de las personas con capacidad intelectual límite, sus principales puntos de reflexión se suman a la propia visión de las personas con capacidad intelectual límite y sus familias:

- La insuficiencia de los diagnósticos, especialmente en los primeros años, implica un factor de riesgo que influye en la derivación de las personas a entidades que no se ajustan al perfil de la misma o a apoyos que no responden eficazmente a su discapacidad. Explicitan la relevancia de incrementar recursos para la investigación.

- Las familias, ante el desconcierto y la desorientación, deambulan buscando apoyos, recorriendo entidades genéricas y específicas.

- Las organizaciones deben abrirse a dar opciones a la diversidad de situaciones familiares, asumiendo un rol más activo desde el ámbito comunitario, evitando la estigmatización.

- A veces, la salida no son las entidades, que aún carecen de una oferta de apoyos completa y que optan, en muchos casos, por mantener a las personas con capacidad intelectual límite en centros ocupacionales o centros especiales de empleo. Una alternativa para la inclusión social son los apoyos de seguimiento en temas de adaptación social y es relevante, asimismo, la metodología de empleo con apoyo como 
estrategia diferente para acceder al mercado de trabajo ordinario.

- La formación profesional ha de abordarse integralmente. Es importante tanto la parte curricular específica y académica, como también la globalidad del marco competencial y jurídico definitorio del entorno laboral para facilitar que la persona con capacidad intelectual límite pueda acceder a un empleo de forma más eficaz e inclusiva.

- $\quad$ El hecho de que muchas veces vaya asociada la discapacidad a problemas de salud mental o conductas desadaptativas como consecuencia del consumo de sustancias tóxicas, requiere del diseño e implementación de programas de prevención en la escuela primaria y secundaria.

- $\quad$ El eje central, según los profesionales, ha de incorporar una estrategia en la que el foco son los apoyos y una intervención individualizada, respondiendo al enfoque de la planificación centrada en la persona.

Es importante, por otro lado, resaltar la necesaria flexibilización organizacional, con programas compartidos entre diferentes organizaciones y con una mayor interacción con otros recursos comunitarios, ya que no existe un especialista único que abarque toda la problemática de la persona con capacidad intelectual límite. Es clave la complementariedad de servicios especializados, integrando además la participación activa de la familia. Es necesario sensibilizar e involucrar también al entorno social para facilitarles la elección de amistades y grupos sociales de referencia, entre otros. El fin último es la prevención del aislamiento social y el impacto psicológico de este aislamiento en las personas con capacidad intelectual límite.

Destacan las necesidades de apoyos en los diferentes ámbitos, especialmente la necesidad de adaptación de los mismos y la relevancia de buscar alternativas metodológicas eficaces y pertinentes al perfil de las personas con capacidad intelectual límite, que sensibilicen al espacio social público y privado para una inclusión social y una vida independiente.

El ámbito laboral es el que señalan como el que comporta mayores factores de riesgo y de prevención. Precisamente es uno de los ámbitos que favorecen la transición a la vida adulta, siendo fundamental la sensibilización de la sociedad.

Se percibe, al igual que las familias y las personas con capacidad intelectual límite, la etapa adolescente como un factor de riesgo en el proceso de inclusión social. Los adolescentes con capacidad intelectual límite, según datos estadísticos, suelen tener mayor fracaso académico, siendo más frecuente la vuelta a servicios especializados educativos no inclusivos. Las relaciones sociales, en estos casos, se complican. Las personas con capacidad intelectual límite sufren mayor rechazo, soledad y proximidad hacia grupos conflictivos.

La familia sigue siendo el agente principal para un cambio personal positivo y, al mismo tiempo, un factor relevante para la autonomía personal. La potenciación de mayor autodeterminación, el uso del ocio inclusivo y la apuesta por los apoyos favorecen la inclusión social de las personas con capacidad intelectual límite.

\section{Conclusiones}

Del discurso transversal de todos los actores relevantes que promueven la inclusión social de las personas con capacidad intelectual límite, se extraen puntos comunes que refuerzan y definen el sentido estratégico compartido de cómo abordar la situación y necesidades de las mismas, cuye eje principal son el nivel y tipo de apoyos.

En este discurso emergen los diferentes factores o dimensiones de una realidad social que es necesario transformar para visibilizar a las personas con capacidad intelectual límite como 
sujetos activos y ciudadanos de pleno derecho. El modo cómo las personas con capacidad intelectual límite expresan y describen sus deseos y expectativas en las diferentes etapas del ciclo vital, especialmente en la edad adulta, hace aún más relevante el modelo social de vida independiente con los apoyos pertinentes.

El contexto familiar, la actitud y rol de la familia es un factor de prevención clave para el desarrollo de las personas con capacidad intelectual límite. Sobre todo, desde la óptica de que puedan optar a una vida autónoma e independiente, tomando como referencia el enfoque de calidad de vida.

Los diferentes actores que intervienen o con los que interactúan las personas con capacidad intelectual límite, tales como la familia, los grupos de iguales, los profesionales u otros, durante su proceso de socialización, contribuyen, tanto para limitar o como para promover su participación en la vida de la comunidad.

Los soportes y servicios que ofrecen las propias entidades representativas y los recursos públicos disponibles son considerados pertinentes, al mismo tiempo que son imprescindibles para incrementar el desarrollo personal y social de las personas con capacidad intelectual límite.

Se ha constatado insuficiente disponibilidad de recursos públicos y administrativos para facilitar un diagnóstico clarificador y temprano. Los diagnósticos imprecisos son, por tanto, una dificultad más a la que hacen referencia las familias y profesionales. Esta indefinición repercute en el acceso a los apoyos oportunos en una etapa de la vida fundamental. A esto se une la dificultad de visibilizarlas, por no tener rasgos evidentes, que en muchos casos hace aún más difícil detectar rápidamente la situación de capacidad intelectual límite.

La deambulación de las familias y la movilidad de unos centros educativos a otros es la tónica repetitiva ya en su etapa educativa, buscando aquellos que le ofrecen apoyos más ajustados a sus necesidades. La insuficiente formación y conocimientos por parte del profesorado de la discapacidad, como un factor de riesgo expresado, dificulta aún más establecer un itinerario de apoyos educativos y adaptativos precisos. La importancia de un enfoque de apoyos integrales que vaya más allá de lo académico, incluyendo programas dirigidos a las habilidades de adaptación deficitarias, favorecería sus transiciones en el ámbito educativo y en su desarrollo personal y social. No obstante, esto requiere financiación y recursos profesionales formados específicamente, lo cual representa una limitación real para la puesta en marcha de estas iniciativas.

Se insiste en que el modelo educativo actual es, en parte inclusivo e integrador, y en parte carente de los suficientes apoyos para abordar la diversidad de necesidades educativas. En este modelo, las personas apuran su estadía, atendidos en algunos casos y desatendidos en otros, aprendiendo dentro de un marco curricular que no contempla sus dificultades de aprendizaje y donde, además, la relación con los iguales es desigual por su diferente ritmo de desarrollo. Los apoyos suelen centrarse en la dimensión educativa académica, limitándose la potenciación y uso de otros apoyos fundamentales, como aquellos que favorecen funciones adaptativas en las personas con capacidad intelectual límite para interactuar de una forma positiva y preventiva con su entorno.

El paso de la educación a la formación profesional es otro vacío expresado principalmente por los profesionales y familiares. La formación ocupacional debería brindar opciones formativas vocacionales adaptadas a las personas con inteligencia límite y muy vinculadas con la dinámica del mercado laboral.

En el plano laboral, aunque, señalan que los agentes económicos, las empresas, y la sociedad en general están más sensibilizados en la importancia de brindar oportunidades de empleo a las personas con capacidad intelectual límite, es imprescindible una mayor coordinación con los agentes sociales, tales como las entidades representativas. De forma 
complementaria, se explicita la importancia de promover un cambio en la legislación actual, que impulse el empleo. Un ejemplo de práctica exitosa para lograr la inclusión laboral, que refuerza lo anterior, es la modalidad del empleo con apoyo, alternativa que abre el espacio laboral ordinario con los apoyos naturales a las personas con discapacidad.

Respecto al tejido asociativo, algunas asociaciones asumen la atención de discapacidades diversas, incluyendo a las personas con capacidad intelectual límite como un perfil más, con apoyos generales no acordes a sus necesidades. Por otro lado, nos encontramos con entidades sociales específicas, con un enfoque que mezcla un modelo más tradicional de atención con un enfoque interactivo y abierto a la comunidad. Los apoyos, en este último caso, son concebidos como herramientas de participación activa de las personas con capacidad intelectual límite en el medio social. Ofrecen servicios, recursos o apoyos dirigidos a cubrir los vacíos sistémicos oficiales o para acceder a ámbitos sociales normalizados que representan otras expectativas personales y sociales de las personas con capacidad intelectual límite.

Surgen programas diversos, unos más protectores y otros de carácter más preventivo, cuya financiación se consigue por vía privada o subvencionada, pero con una exigencia práctica, por parte de la Administración Pública, de entender el apoyo que se brinda a través de estos servicios como un coste contable de plaza y no como un apoyo continuo y permanente, en muchos casos. Los profesionales, ligados a estas entidades con los apoyos que éstas logran financiar, trabajan con las familias y con las personas con capacidad intelectual límite, pero requieren de mayores recursos para hacer real la inclusión social.

Desde el punto de vista de los profesionales y las familias, tanto la Administración Pública como las políticas públicas, la normativa actual y los recursos oficialmente disponibles no responden a las diversas necesidades de las personas con capacidad intelectual límite. La Administración
Pública ha de actuar de forma más activa y ser garante del equilibrio y distribución de los apoyos para una vida independiente, siempre bajo el enfoque de calidad de vida.

Un vacío identificado por las familias y profesionales son aquellos programas de apoyo para las personas con conductas disruptivas (víctimas potenciales de un entorno social desestructurado, situaciones delictivas o consumo de drogas), que previnieran posibles penalizaciones legales, con las consecuencias que conllevan (concretamente una situación de riesgo de exclusión social permanente).

Otros factores de riesgo, además del problema de financiación, son el aislamiento geográfico para acceder a determinados apoyos, la situación socioeconómica y el contexto desestructurado familiar.

En todos los ámbitos mencionados, los apoyos son centrales en todas las etapas vitales de las personas con capacidad intelectual límite, sobre todo en la infancia y la adolescencia. En este sentido, es clave potenciar la investigación, que permita entender mejor sus necesidades y tipos de apoyos en cada momento, especialmente en la detección a tiempo de la situación de discapacidad, accediendo lo antes posible a los apoyos, como por ejemplo el Servicio de Atención temprana, pero recordando asimismo que no es el objetivo último etiquetar y separar, sino para favorecer los apoyos correspondientes. Esto, a su vez, hace imprescindible que los apoyos formales e informales en la escuela, en la formación profesional o vocacional, en el empleo, en la formación para la autonomía personal y vida independiente, y en general, se adapten o se trabajen conforme a la situación de la personas, conforme la entendimiento de sus necesidades adaptativas, y no sólo en base al criterio de los límites de la inteligencia. Se apuesta, por tanto, por un enfoque o modelo social de intervención activo, abierto a una comunidad que esté sensibilizada respecto a las diferencias y diversidad de estas personas. Un modelo, además, en el que las entidades sociales ofrezcan apoyos no institucionalizados, como meros referentes ante determinada situación, 
tanto para las familias como para las personas con capacidad intelectual límite.

Los apoyos considerados naturales (familia, amigos, hermanos) y los formales han de asumir un rol de soporte, que vayan en la dirección de ir desapareciendo a medida que las personas van avanzando en su itinerario de vida independiente, como recalcan los profesionales. El rol de las asociaciones, como apoyo complementario, es relevante para el acceso a los grupos de iguales y promover la autonomía personal y social.

Es importante conocer el entorno social y cuáles son las variables externas necesarias para hacerlo más accesible, considerando la vulnerabilidad de las personas con capacidad intelectual límite por las dificultades asociadas a las habilidades sociales y comunicativas ante el entorno social, cultural, laboral o político inmediato.

La sensibilización de la sociedad respecto a los derechos de las personas con capacidad intelectual límite es fundamental y tiene que tender a que las personas con capacidad intelectual límite pueden aportar por ellos mismos a través de una participación activa en la defensa de dichos derechos, como práctica habitual que se viene realizando desde la figura del autogestor.

Aunque no se explicitan abiertamente las diferencias de género, sí que comentan los diferentes riesgos en hombres y mujeres con capacidad intelectual límite ante determinadas situaciones de abusos y discriminación social. También se hace evidente en los roles aprendidos en sus entornos naturales y en los diferentes ámbitos de la sociedad.

En línea con el modelo de intervención que se propone, dentro de las buenas prácticas relatadas por los profesionales, familias, personas con capacidad intelectual límite e investigadores, se muestra precisamente la estrategia de trabajar con las personas con capacidad intelectual límite en su entorno natural, en interacción permanente con la comunidad, donde los apoyos van pasando de ser extensos a intermitentes e incluso a desaparecer. En este caso, la complementariedad y la coordinación de apoyos son fundamentales, además de la implicación de las familias y entidades asociativas. Uno de los ámbitos más desarrollado es el ocio inclusivo. Es un factor preventivo más hacia una inclusión social efectiva en la comunidad.

\subsection{Propuestas de actuación}

Los resultados, el análisis de la información y el mismo discurso compartido por los referentes claves de este estudio, aportan propuestas para orientar actuaciones diversas en diferentes ámbitos, con la finalidad de mejorar la calidad de vida de las personas con capacidad intelectual límite y de transformar la realidad social actual positivamente hacia una realidad inclusiva y el cumplimiento del deseo de una vida autónoma e independiente: Entre estas propuestas destacan:

\section{- Cambios y ajustes de la normativa actual}

Modificación de instrumentos de reconocimiento de derechos (Certificado de Discapacidad, Sistema de Dependencia) que impulse el acceso, en igualdad de oportunidades, a determinados servicios y recursos como establece la Convención de Naciones Unidas sobre los derechos de las personas con discapacidad.

Modificar la condición jurídica, el límite del $33 \%$, requerida para ser sujeto de medidas de discriminación positiva y de incentivación a la contratación laboral.

Asesoramiento especializado sobre las personas con capacidad intelectual límite para la elaboración de normativa legal.

Seguimiento de la aplicación de la Convención sobre los derechos de las personas con discapacidad en la mejora de la vida cotidiana de las personas con capacidad intelectual límite y sus familias. 
- Políticas sociales inclusivas (educación y empleo)

Priorizar la educación inclusiva y el empleo con apoyo como modalidades eficaces en la inclusión social de las personas con capacidad intelectual límite.

Formación de los profesionales y adaptación del sistema educativo (metodología, contenidos, etc.) a las necesidades específicas de las personas con capacidad intelectual límite.

Evaluación de la capacidad de respuesta del sistema educativo a las demandas de las personas con capacidad intelectual límite, incorporando los ajustes razonables y aumentando, al mismo tiempo, las oportunidades de desarrollo profesional.

Establecimiento de medidas que incentiven el empleo de las personas con capacidad intelectual límite en el mercado de trabajo normalizado, con las adaptaciones razonables para que puedan disfrutar de una vida independiente y autónoma.

Aumento de los presupuestos en las políticas sociales destinadas a las personas con capacidad intelectual límite, sobre todo de carácter preventivo.

- Servicios con respuestas específicas e inclusivas

Diseñar programas de carácter preventivo que incluyan aspectos personales, sociales (entornos con riesgos de abusos y conductas delictivas) y enfermedad mental, entre otros contenidos.

Mejorar el sistema sociosanitario, para que facilite una atención acorde a las necesidades particulares de las personas con capacidad intelectual límite.

Potenciar la capacidad de toma de decisiones como personas que viven con normalidad en sus entornos sociales inmediatos y con una participación activa en la defensa de sus propios derechos.

Cambio de perspectiva en el rol que han de cumplir las asociaciones. Este rol debe responder a un rol de apoyo puntual, centrado en la participación de las personas con capacidad intelectual límite en la comunidad, más que como un ente protector.

\section{- Apoyos naturales y tejido social}

Existencia de un tejido asociativo que contemple una oferta integral de servicios y apoyos coordinados y con la participación activa de familias, personas con capacidad intelectual límite y profesionales.

Formación y orientación a las familias en todas las etapas del ciclo vital de las personas con discapacidad y de la propia familia, especialmente la transición a la vida adulta, apostando por diferentes modalidades formativas, como por ejemplo escuelas de padres.

Generalizar apoyos naturales y el equilibrio de los mismos, con el objetivo final de que las personas con capacidad intelectual límite opten a una vida autónoma e independiente.

Potenciar la capacidad de autogestión y toma de decisiones en todos los ámbitos de la vida, como ciudadanos de pleno derecho, es relevante para la inclusión social.

Coordinación de todos los actores involucrados en el proceso de desarrollo personal y social de la persona: profesionales, familias, administración, entidades, etc.

- Planificación centrada en la persona y desde un enfoque de calidad de vida 
Hacer de los itinerarios individualizados y con el enfoque de la planificación centrada en la persona el eje de los modelos de intervención con las personas con capacidad intelectual límite.

Demostrar una mayor responsabilidad política, promoviendo políticas con mayor peso de discriminación positiva en favor del colectivo de personas con capacidad intelectual límite, además de proponer asuntos relativos a las mismas que dependan exclusivamente del Estado y no estén externalizados a otro tipo de entidades.

Facilitar modelos de viviendas alternativas para personas con capacidad intelectual límite con un nivel de autonomía aceptable y con expectativas de optar por una vida independiente. 


\section{Referencias bibliográficas}

Artigas-Pallarés, J. (20I I): "Inteligencia y funcionamiento intelectual límite”, en ArtigasPallarés, J. y Narbona, J. (eds.): Trastornos del neurodesarrollo. Barcelona: Viguera Editores.

Artigas-Pallarés, J. et al. (2007): "Relación entre capacidad de inteligencia límite y trastornos del neurodesarrollo”. Revista de neurología, 44 (I 2): 739-744.

Denzin, N. (1989): Strategies of multiple triangulation. The research Act: A theoretical introduction to sociological methods, Nueva York: McGraw Hill.

Egido, I. et al. (2009): “La inclusión social y laboral de las personas con discapacidad intelectual mediante los programas de empleo con apoyo. Un reto para la orientación". Revista Española de Orientación y Psicopedagogía, 20 (2): I35-I46.

España. Ley 26/20I I, de I de agosto, de adaptación normativa a la Convención Internacional sobre los Derechos de las Personas con Discapacidad, Boletín Oficial del Estado, 2 de agosto de 20 I I, núm. I 84, pp. $87478-87494$.

Frontera. M y Gómez Bahillo, C. (20I3): Inteligencia Limite, Perfil, necesidades, recursos, $y$ propuestas de mejora, Zaragoza: Universidad de Zaragoza y Asociación EnLínea.

Giné, C. (2006): “Aportaciones a la comprensión de las personas con inteligencia límite. Riesgos y perspectivas, en Delgado, M. y Gallén, C. (coords.): Normalidad y límite: construcción e integración social del «borderline»". Madrid: Centro de Estudios Ramón Areces.

Laparra, M. et al. (2007): "Una propuesta de consenso sobre el concepto de exclusión social. Implicaciones metodológicas”. Revista Española del Tercer Sector, 5: I 5-57.

Huete, A. et al. (20I 5): Estudio sobre la situación de las personas con capacidad intelectual limite, Madrid: Ministerio de Sanidad, Servicios Sociales e Igualdad.

Pallisera, M. et al. (2013): "Transición a la vida adulta de jóvenes con discapacidad intelectual, la opinión de los profesionales de servicios escolares y postescolares". Revista Española de Orientación y Psicopedagogía, 24 (2): IOO-I I 5.

Palos, N. (2002): Personas con Inteligencia límite: Un colectivo en tierra de nadie (en línea). <http://www.pasoapaso.com.ve/index.php/ temas/discapacidad/discapacidad-intelectual/ inteligencia-limite/item/I 220-personas-coninteligencia-1\% $\mathrm{C}_{3} \%$ ADmite-un-colectivo-entierra-de-nadie>, acceso 2 I de junio de 2016.

Riera, C. (dir.) (2003): Personas con discapacidad intelectual y necesidad de apoyos intermitentes: situación, necesidades y demandas. Una aproximación a la población con inteligencia limite-ligera, Madrid: Observatorio de la Discapacidad.

Collet, M. et al. (2004): Aproximación sociológica a las personas con DINAI (en línea). <http:// sid.usal.es/idocs/F8/8.2.I.2-I39/I45/I3_34_ dossier.pdf $>$, acceso 2 I de junio de 2016 . 